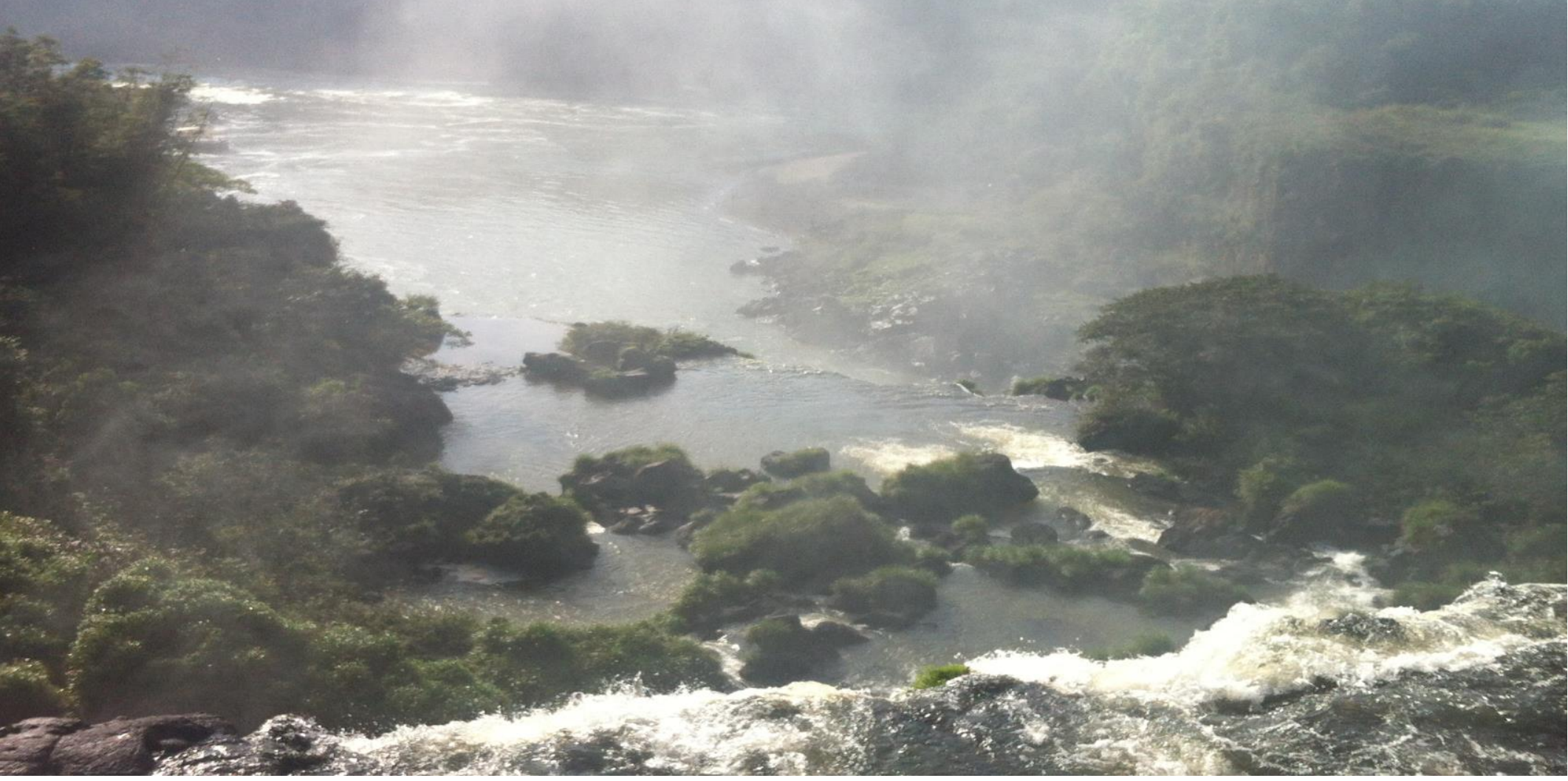

\title{
Susceptibilidad a erosión hídrica y procesos gravitacionales en la subcuenca de alta montaña del río Jampa, Veracruz, México
}

\section{Oswaldo Trinidad Hernández - Katrin Sieron - Francisco Córdoba Montiel}

RESUMEN: En el presente trabajo se determinó la susceptibilidad actual a procesos de erosión hídrica y procesos gravitacionales en la subcuenca de alta montaña del río Jamapa en el flanco Norte del volcán Citlaltépetl o Pico de Orizaba. El estudio se llevó a cabo mediante el empleo de Sistemas de Información Geográfica (SIG), con la aplicación de los modelos ERU y Mora-Vahrson. Los resultados mostraron una concordancia de ambos modelos de acuerdo con la distribución de los mayores grados de susceptibilidad. Contrario a lo que se esperaba, los modelos arrojaron mayores susceptibilidades tanto de erosión hídrica como a procesos gravitacionales en la parte baja y media de la subcuenca, lo cual se debe a que los principales activadores hídricos se presentan con mayores índices en las partes bajas y disminuyen con la altitud.

Palabras clave: Erosión hídrica; remoción en masa; susceptibilidad; Citlaltépetl (Pico de Orizaba); Sistemas de Información Geográfica. 
Artículo - Observatorio Sismológico y Vulcanológico

\begin{abstract}
In the present work, the current susceptibility to water erosion- and gravitational processes in the high mountain sub-basin of the Jamapa River on the North flank of the Citlaltépetl or Pico de Orizaba volcano was determined. The study was carried out through the employment of Geographic Information Systems (GIS), with the application of the ERU and Mora-Vahrson models. The results show a concordance of both models according to the distribution of the highest degrees of susceptibility. Contrary to what was expected, the models show a greater susceptibility to both water erosion and gravitational processes in the lower and middle part of the sub-basin, which is due to the fact that the main water activators are presented with higher rates in the lower parts and decrease with altitude.
\end{abstract}

Keywords: Water Erosion; Mass Removal; Susceptibility; Citlaltépetl (Pico de Orizaba); Geographic Information Systems.

\title{
Introducción
}

$\mathrm{E}$

1 volcán Pico de Orizaba está localizado en el extremo este del Cinturón Volcánico Mexicano (CVM) y cuenta con una altitud de 5675 m s. n. m. (Zimbelman et al., 2004), por lo que representa la mayor elevación del territorio. Como resultado de la altitud, las pronunciadas pendientes y características climatológicas asociadas, se generan notables procesos físicos, los cuales son de gran interés para ser estudiados.

En el caso del presente trabajo, se analizan la susceptibilidad a procesos erosivos hídricos y por gravedad que se desarrollan en la cara noreste de dicho volcán. El área de estudio ha sido delimitada, utilizando la traza de la subcuenca de alta montaña del río Jamapa. En cuanto a altitud se refiere, la parte más baja de la subcuenca se encuentra a $1490 \mathrm{~m} \mathrm{~s}$. n. m., mientras que la parte más alta se presenta a los $5500 \mathrm{~m} \mathrm{s.} \mathrm{n.} \mathrm{m.,} \mathrm{ya} \mathrm{bajo} \mathrm{la} \mathrm{influencia} \mathrm{del}$ glaciar Jamapa. En el caso de la subcuenca Jamapa, la variación de climas presentes van desde semicálido húmedo (A)C(fm), hasta el frío de alta montaña E(T)H (INEGI, 2010).

Actualmente, no existen registros acerca de la intensidad en que se presenta la erosión en la subcuenca de alta montaña del río Jamapa y en qué grado las zonas susceptibles a erosión ya coinciden con zonas susceptibles a remoción en masa, correspondiente a una evolución de los procesos de inestabilidad de ladera y por ende íntimamente relacionados. Debido a 
esto se plantea la necesidad de determinar los niveles de susceptibilidad de erosión y de remoción en masa, así como su distribución espacial determinada por los factores que intervienen en el proceso.

\section{Área de estudio}

El área de estudio se encuentra localizada en el flanco noreste del volcán Pico de Orizaba (Figura 1). Su elección se apegó al área de estudio abarcada por el proyecto "Belowground ecosystem services in plant communities a long elevational gradients in France and Mexico - ECOPICS" (financiado por CONACYT (México) y ANR (Francia)); sin embargo, para este estudio, la delimitación del área de trabajo se extendió a las dimensiones de la subcuenca del

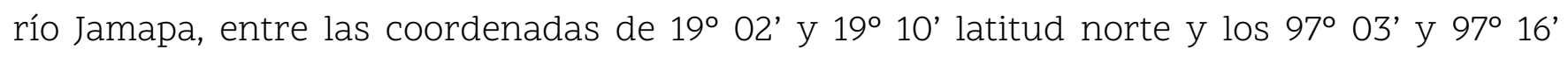
latitud oeste. Esta elección fue tomada debido al estudio del factor hídrico y la dinámica que presentan los canales de desagüe en el flanco noreste.

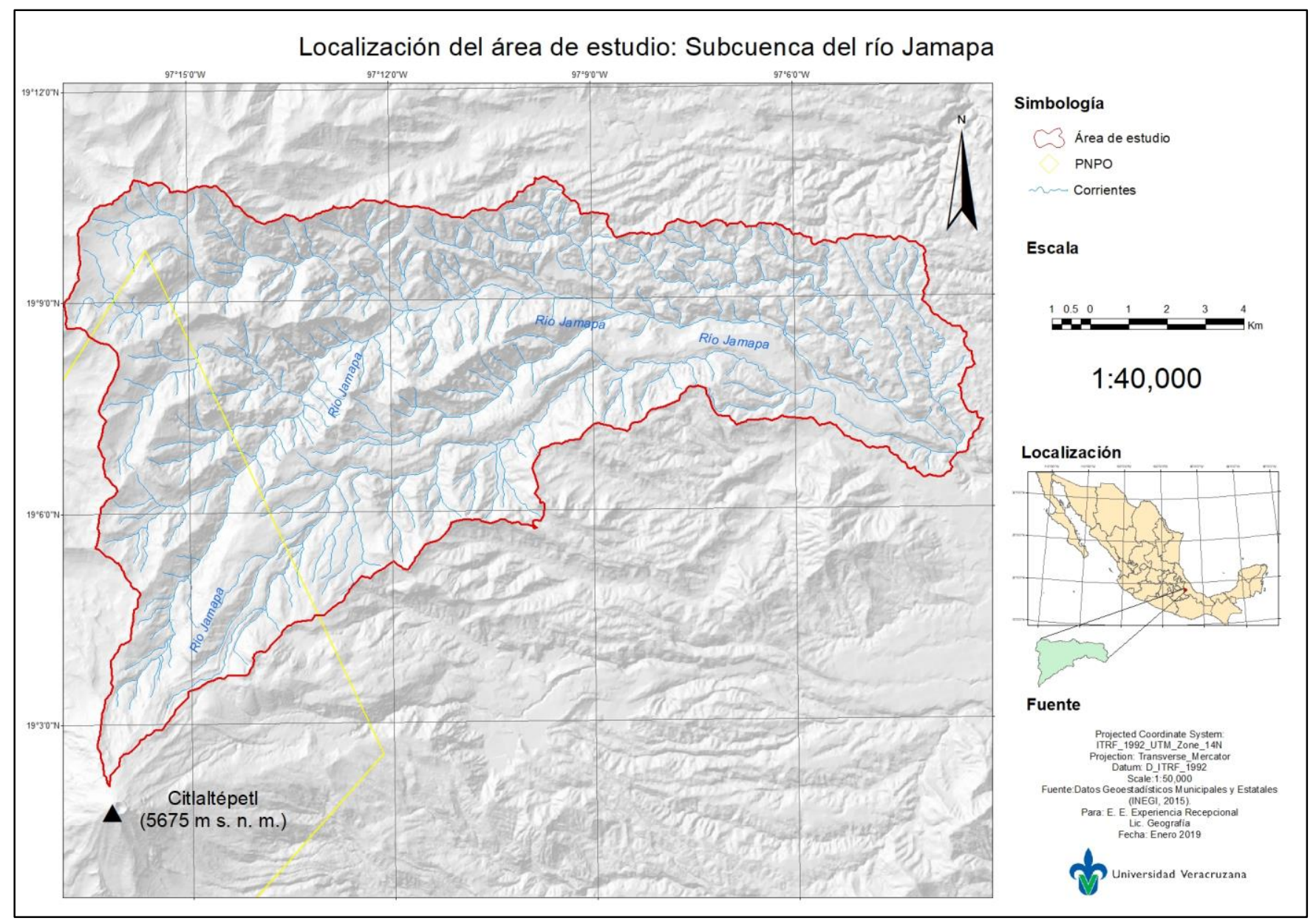

Figura 1. Subcuenca de alta montaña del río Jamapa

UVserva No. 8 Octubre 2019 - marzo 2020, ISSN: 2448-7430. 
Artículo - Observatorio Sismológico y Vulcanológico

\section{Metodología y marco teórico}

Los insumos para la reproducción de las variables propuestas por los dos modelos utilizados (Susceptibilidad hídrica y susceptibilidad gravitacional), así como para la caracterización del área de estudio fueron obtenidos de distintas dependencias gubernamentales y no gubernamentales. Del Instituto Nacional de Estadística, Geografía e Informática, fueron descargados los datos topográficos e hidrográficos contenidos en la carta del área de estudio: E14B46 (INEGI, 2015). Los datos de uso de suelo fueron obtenidos de la Comisión Nacional Forestal (CONAFOR, 2013). Los datos geológicos y litológicos fueron extraídos de la Carta Geológica Minera del Servicio Geológico Nacional y del mapa geológico del volcán publicado por Macías (2005). Las bases de datos de las estaciones seleccionadas para el cálculo de agresividad pluvial fueron descargadas del Servicio Meteorológico Nacional (SMN, 2018) y, por último, las imágenes satelitales bajo licencia del satélite Spot 5 fueron proporcionadas por la Coordinación Universitaria de Observatorios (CUO) de la Universidad Veracruzana.

Para determinar la susceptibilidad hídrica en la subcuenca del río Jamapa fueron aplicados tanto los factores, como las categorías asignadas por los autores que desarrollaron el modelo ERU (Märker et al., 2001). En el caso de los factores requeridos, responden a la interacción y característica propia de cada uno de ellos en los procesos erosivos hídricos. Los primeros tres factores que se presentan a continuación son parte de las condiciones propias de la cuenca, mientras que el último factor es detonador del proceso erosivo hídrico:

Parámetros morfológicos y fisiográficos. Este factor se encuentra relacionado con la morfología de la pendiente y las características del terreno. En el sistema SIG estos parámetros son generados mediante un Modelo Digital de Elevación (MDE), a partir del cual se determinaron las variables de Curvatura de la Pendiente (Blaga, 2012), Grado de la Pendiente (Mora-Vahrson, 1991) y Longitud de la Pendiente (Lorentz y Schulze, 1995). Posteriormente para la determinación del índice de susceptibilidad fisiográfica del terreno se multiplicaron entre sí los tres rásters generados anteriormente (Longitud de la pendiente, curvatura de la pendiente y grados de la pendiente) mediante una herramienta de cálculos de ráster y siguiendo la clasificación propuesta por los autores del modelo (Flügel, et al., 2001).

Erodabilidad litológica. En el presente caso, la forma de determinar el factor fue tomada a partir del factor Sl (Susceptibilidad litológica) del modelo Mora-Vahrson, por lo cual el procedimiento será explicado en el modelo siguiente. La elección de este factor para este 
modelo se basa en los supuestos de que no sólo influyen las características de cada una de las rocas, sino que también la edad, ya que ambas determinan los niveles de meteorización.

Densidad de cobertura vegetal. Este facor se genero mediante la creación del índice NDVI a partir de fotografías digitales (Gilabert et al. 1997; Meneses-Tovar, 2011). La clasificación de cobertura vegetal fue de acuerdo con la propuesta por Van Zuidam (1985).

Agresividad climática. La obtención del factor detonante se realizó a partir del modelo IMF (Arnoldus, 1977). Los índices se determinaron mediante los datos diarios de 17 estaciones en un periodo de 40 años. Dichos datos fueron tomados del Servicio Meteorológico Nacional (2018). Una vez calculados los índices de agresividad climática de cada estación, estos fueron vectorizados e interpolados en formato ráster.

Finalmente, una vez determinados cada uno de los factores utilizados para el modelo, los rásters de cada factor fueron multiplicados entre sí mismos y posteriormente reclasificados de acuerdo a las categorías determinadas por Märker et al. (2001). Los pasos seguidos mediante el procesamiento de los factores fueron sintetizados y se presentan en la figura 2.

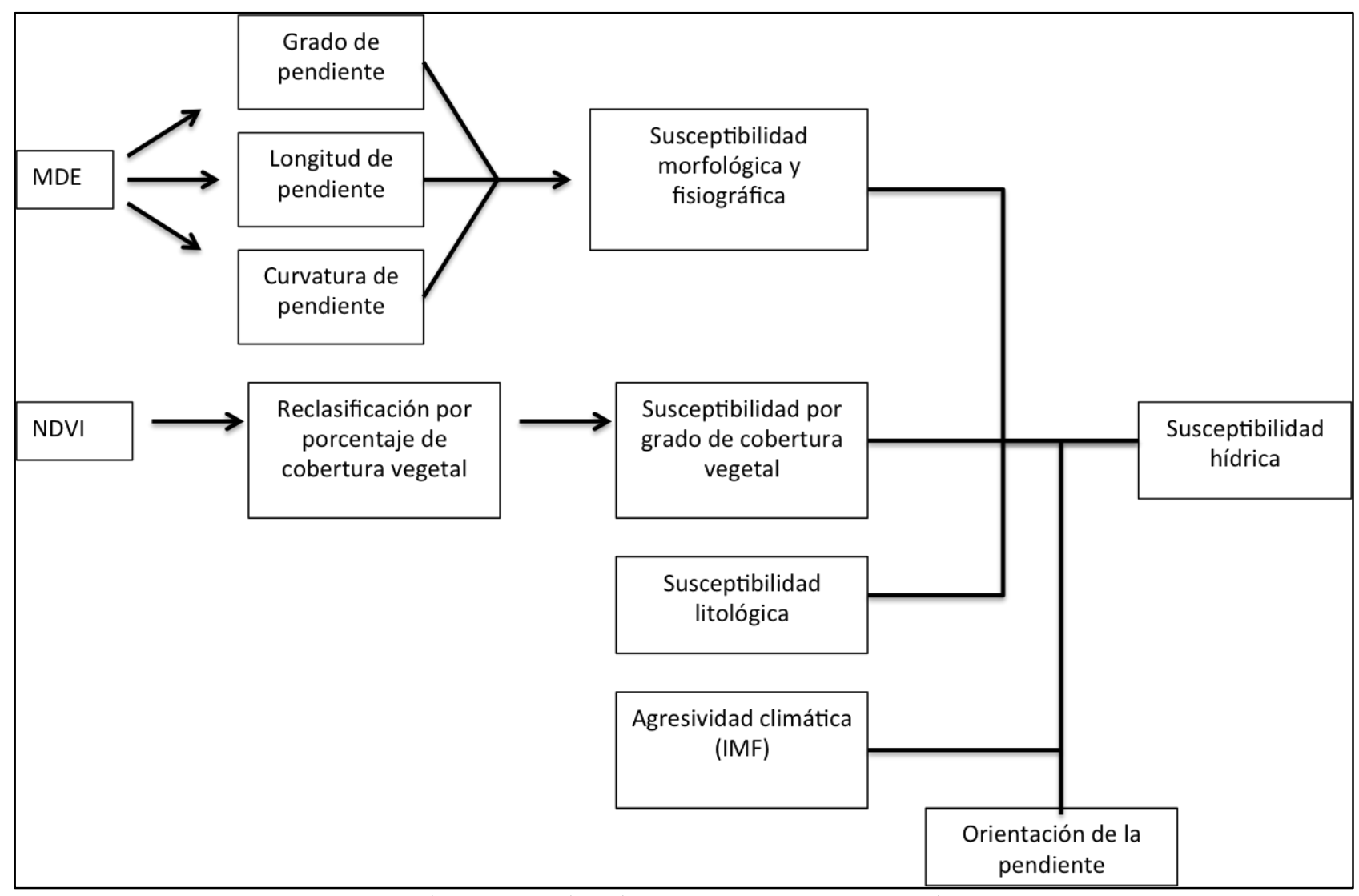

Figura 2. Proceso de determinación de erosión hídrica (modificado después de Märker et al., 2001).

UVserva No. 8 Octubre 2019 - marzo 2020, ISSN: 2448-7430. 
Artículo - Observatorio Sismológico y Vulcanológico

Para el desarrollo del método Mora-Vahrson es necesaria la obtención de cinco factores, los cuales interfieren en la morfodinámica de los procesos de remoción en masa. Dichos factores se clasifican en dos grupos, por un lado, se encuentran los factores intrínsecos o de susceptibilidad del terreno, como lo son el relieve relativo (Sr), las características de la litología (Sl) y la humedad del suelo (Sh), y por otro los factores externos o de disparo como lo son la actividad sísmica (Ts) y los niveles de precipitación (Tp) (Mora et al., 1992).

La distribución espacial de los datos en cuanto a los niveles de cada factor hace posible su cálculo mediante los Sistemas de Información Geográficos (SIG's), ya que cada celda contiene un valor. Para la determinación de los índices de susceptibilidad en los rásters se utiliza la siguiente ecuación (Mora y Vahrson, 1994):

$$
H=I T R I N * D I S P
$$

Donde $H$ es el grado de susceptibilidad a remoción en masa, INTRIN son valores producto de la combinación de elementos intrínsecos o pasivos y DISP el valor de los factores de disparo:

$$
H=(S r * S h * S l) *(T s+T p)
$$

La obtención de cada una de las variables de la ecuación y su respectivo procesamiento en SIG se realizó de la siguiente manera:

Susceptibilidad del relieve (Sr). En el caso de la susceptibilidad del relieve, se hizo uso del $D E M$ utilizado en el modelo anterior. El DEM fue sometido al procesamiento de pendientes, donde posteriormente fue reclasificado de acuerdo con seis niveles de susceptibilidad, dependiendo del grado de pendiente (IFGRN, 2002)

Las capas de la litología del área de estudio en formato shape fueron obtenidas mediante la digitalización del mapa detallado publicado por Macías (2005) y modificado por sieron (2018), así mismo, en las zonas no cubiertas por ambos autores se hizo uso de la geología presente en la Carta Geológica Minera del Servicio Geológico Mexicano con la clave E14 perteneciente al estado de Veracruz y Puebla (SGM, 2002). Los rangos de susceptibilidad litológica fueron asignados a cada clase de roca según los parámetros establecidos por los autores Mora y Vahrson (1994), los cuales dependen de la influencia de los tipos de materiales y rocas en la activación de los procesos de remoción en masa (Quesada y Feoli, 2018). 
Humedad del suelo (Sh). Para la determinación de la unidad del suelo, igualmente se hizo uso de las estaciones mencionadas en el modelo anterior. A partir de los datos de precipitación se generó el promedio mensual por estación a lo largo del periodo de 40 años. Estos promedios mensuales fueron vectorizados en formato raster por cada uno de los meses, y se reclasificaron de acuerdo a la metodología del modelo Mora-Vahrson (Mora y Vahrson, 1994).

La clasificación en cuanto a la intensidad de los sismos para propiciar movimientos de material se realiza a partir de las intensidades en la escala de Mercalli Modificada (MM), digitalizándolas a partir del mapa de intensidades de México (CENAPRED, 2001). La digitalización se llevó a cabo en Google Earth y el formato kmz fue convertido a formato shp y posteriormente a ráster. Dependiendo de la intensidad sísmica de cada zona, se le fue asignando el valor correspondiente determinado en el modelo Mora-Vahrson (Mora y Vahrson, 1994).

Para la obtención del factor detonante por precipitación se hizo uso de la misma base de datos utilizada para la obtención del factor Sh. Dentro del mismo periodo de 40 años se determinó la precipitación máxima ocurrida registrada por cada una de las estaciones. Este valor fue asignado a los promedios mensuales en el momento de la vectorización. Posteriormente se generó una interpolación de la variable de precipitación máxima mediante el valor de cada una de las estaciones.

El ráster de interpolación fue reclasificado posteriormente en 5 rangos. Valores menores a 100 recibieron una categorización de muy bajo y con un valor asignado de 1, de 100 a 200 con calificación de bajo con valor de 2, de 200 a 300 con cualificación medio y valor de 3, de 300 a 400 con categoría alto y valor de 4 y, por último, valores mayores a 400 con categoría de muy alto y un valor de 5.

Una vez determinados los 5 factores en formato ráster, se procedió a realizar el cálculo de la susceptibilidad del área de estudio. Para realizar dicho cálculo en los SIG es necesario basarse en la fórmula propuesta por Mora y Vahrson, explicada con anterioridad. Finalmente, el ráster de susceptibilidad de procesos de remoción en masa (H) fue sometido a una reclasificación de acuerdo con los parámetros determinados por los autores del modelo (Mora y Vahrson, 1994). 
Artículo - Observatorio Sismológico y Vulcanológico

\section{Resultados}

Susceptibilidad a erosión hídrica

De acuerdo con los resultados del modelo (Figura 3E), la susceptibilidad con la mayor distribución a lo largo de la cuenca es la Moderadamente ligera con 48\%. En la zona oeste y sur oeste de la cuenca su presencia es explicada por la baja agresividad de las precipitaciones, mientras que en la parte norte es determinada por la longitud de la pendiente media, por orientación de las pendientes hacia el sur y oeste y por una susceptibilidad litológica y de cobertura vegetal Moderada. En la zona centro y este se presenta en pendientes orientadas al sur, oeste y en pocos casos al norte, con pendientes ligeras a moderadas.

La segunda unidad con mayor presencia es la susceptibilidad moderada (25\%), con mayor distribución en la zona central y noroeste, donde se presentan mayores patrones de drenaje (cauces) y una concentración de índices elevados de grados de la pendiente. En esta parte de la subcuenca el índice de agresividad es medio, sin embargo, la susceptibilidad a erosión hídrica aumenta en pendientes orientadas hacia el noreste y norte, de donde provienen los eventos de precipitación.

En la parte suroeste también se presenta una susceptibilidad moderada de manera aislada en el cono volcánico y aunque los índices de agresividad climática son bajos la presencia de esta categoría es debida a la existencia del material piroclástico no consolidado en ese lugar y por la escasa vegetación. En la zona sureste, centro y este, así como en pequeñas partes aisladas de la zona noroeste, la intensidad de susceptibilidad hídrica Moderada se encuentra acompañada de una intensidad por susceptibilidad hídrica Severa (9\%), en el caso de las zonas centro, sur y este, es originada por una susceptibilidad media de las unidades litológicas, por la profunda longitud e inclinación de la pendiente que presenta el canal principal del río Jamapa y rangos medios, altos y muy altos de agresividad climática, así como susceptibilidad por densidad vegetal Severa.

En el caso de la zona noroeste la susceptibilidad Moderada se presenta por altas pendientes, por agresividad climática Media, por orientaciones hacia el norte y por susceptibilidad por densidad vegetal Muy severa. Por último, la unidad Ligera y Muy ligera se presenta de manera aislada, con características fisiográficas tales como: poca pendiente, morfología cóncava, orientadas al oeste, con índices de agresividad climática bajo, con susceptibilidad 
litológica Bajo y Moderado y en la mayoría de los casos con susceptibilidad de cobertura vegetal Moderada.



Figura 3. A) Mapa de susceptibilidad fisiográfica. B) Susceptibilidad litológica. C) Susceptibilidad por cobertura vegetal. D) Agresividad climática. E) Mapa general de susceptibilidad a erosión hídrica.

\section{Susceptibilidad a procesos gravitacionales}

De acuerdo con los resultados arrojados por el modelo de susceptibilidad a presentarse procesos gravitacionales o de remoción en masa (Figura 4F), el índice de mayor importancia en cuanto a presencia espacial es el Moderado (64\%), su distribución en la parte alta de la subcuenca está influenciada por pendientes abruptas, las cuales tienen valores de susceptibilidad Media, Alta y Muy Alta y humedad del suelo Muy baja, en este último caso, es la variable que mayor influencia tiene. En cuanto a el área de susceptibilidad Modera presente en la zona centro-este los factores que propician son las pendientes Moderadas a Altas, la susceptibilidad litológica Moderada y la susceptibilidad por actividad sísmica Elevada. 
Artículo - Observatorio Sismológico y Vulcanológico

La segunda unidad con mayor extensión (23\%) y la primera con el nivel potencial más elevado en el área de estudio es el índice de susceptibilidad Medio. Su presencia en la parte extrema este se origina por la susceptibilidad Media de las tobas andesíticas en combinación conjunta de la humedad del suelo Medio, así como por la actividad sísmica elevada. En el sureste su presencia es determinada por la susceptibilidad Media de los depósitos indiferenciados, por la humedad Bajo y Medio del suelo, por actividad sísmica elevada, así como por susceptibilidad de las pendientes Altas y Muy altas presentes en los barrancos del valle principal del río Jamapa. En el caso de la zona aislada del norte es resultado de la actividad sísmica Elevada y de la humedad del suelo Bajo.

Por último, las dos unidades con menor extensión son la susceptibilidad Baja y Muy baja, ambas con 7\% de distribución en la subcuenca. En el caso de la primera su presencia se limita a la zona sur del área de estudio, donde se encuentran los menores valores de susceptibilidad de la mayoría de los factores, como el caso de la distribución de precipitación máxima (Muy bajo), de la pendiente (Media y Moderada), de la susceptibilidad litológica (Bajo), de humedad del suelo (Muy bajo), así como la actividad sísmica Media. En el segundo caso, el índice sólo se limita en zonas aisladas en lugares de pendientes menores a 10\% de inclinación ubicadas en zonas altas y en el fondo de valles.
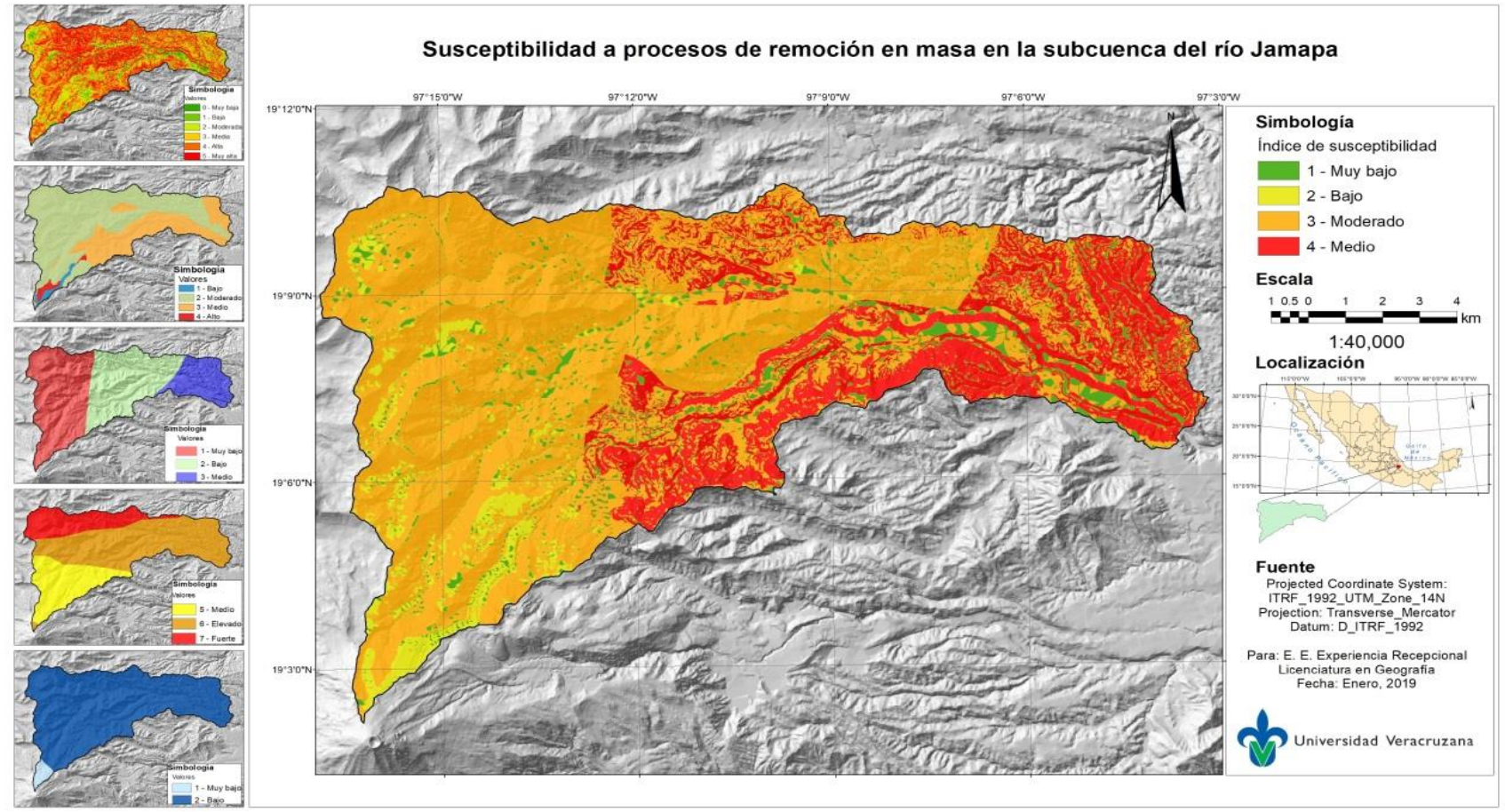

Figura 3 A) Susceptibilidad de la pendiente. B) Susceptibilidad litológica. C) Susceptibilidad por humedad del suelo. D) Intensidad de activación por actividad sísmica. E) Activación por precipitación. F) Mapa general de susceptibilidad a procesos de remoción en masa. 


\section{Conclusión}

En los resultados de ambos modelos se encontró una concordancia en la distribución de las magnitudes de cada modelo. Las mayores susceptibilidades, tanto de erosión hídrica como a procesos de remoción en masa se identificaron en las partes inferiores de la cuenca, donde se presentan los mayores factores activadores y detonantes del área de estudio. Cabe resaltar que estas intensidades no fueron las más altas contempladas en los modelos.

Así mismo, algo singular contrario a lo que se esperaba, es que en las partes altas ambos modelos no arrojan susceptibilidades significativas de erosión a pesar de la falta de cobertura vegetal y de la presencia de unidades litológicas con susceptibilidad alta. Esto puede ser explicado por la falta de precipitación generada por el gradiente altitudinal en grandes altitudes.

De igual forma se observó en las visitas a campo la existencia de altos procesos erosivos y de remoción en masa en la parte alta, lo que lleva a intuir que los factores activadores de los procesos no son necesariamente desencadenados por precipitación, sino eólicos y por los afluentes generados por el derretimiento del glaciar Jamapa. Este flujo de agua, no considerado dentro de los modelos, tiene la capacidad de transportar material regolítico intemperizado y el potencial para desestabilizar la ladera mediante la creación de cárcavas a causa del mismo material suelto.

\section{Referencias}

Arnoldus, H. M. (1977). Methodology used to determine the maximum potential average annual soil loss due to sheet and rill erosion in Morocco. FAO soils bulletin, 34, 39-51.

Blaga, L. (2012). Apects regarding the signifiance of the curvature types and values in the studies of Geomorphometry assisted by GIS. Analele Universitatii din Oradea, 327337.

CENAPRED. (2001). Mapa de intensidades sísmicas de México 1:1,000,000.

CONAFORT. (2013). Conjunto de datos vectoriales de uso de suelo y vegetación del Estado de Veracruz 1:50,000.

Flügel, W. A., Märker, M., \& Rodolfi, G. (2001). Erosion Response Units (ERU): A regional erosion concept for sustainable Integrated Catchment Management and its application in a semi arid catchment in Swaziland, Southern Africa. Dipartamento Scienze del Suelo e Nutrizione della Planta. 
Artículo - Observatorio Sismológico y Vulcanológico

Gilabert, M. A., González-Piquera, J., \& García-Haro, F. J. (1997). Acerca de los índices de vegetación. Revista de Teledetección.

INEGI. (2010). Unidades climatológicas 1: 1,000,000. Datos vectoriales de clima.

INEGI. (2015). E14B46. Carta topográfica 1:50,000.

Instituto Federal de Geociencias y Recursos Naturales. (2002). Mapa de susceptibilidad a deslizamientos de Nicaragua; El método Mora-Vahrson. INETER.

Lorentz, S., \& Schulze, R. (1995). Hydrology and agrohydrology: a text to accompany the ACRU 3.00 agrohydrological modelling system. Water Research Commission, Pretoria.

Macías, J. L. (2005) 'Geología e historia eruptiva de algunos de los grandes volcanes activos de México', Boletín de la Sociedad Geológica Mexicana, 57(3), pp. 379-424.

Märker, M., Moretti, S., \& Rodolfi, G. (january de 2001). Assessment of water erosion processes and dynamics in semi-arid regions of Southern Africa ( Kwazulu / Natal , RSA, and Swaziland) Using the Erosion Response Units Concept. Geografia Fisica e Dinamica Quaternaria.

Meneses-Tovar, C. (2011). El índice normalizado diferencial de la vegetación como indicador de la degradación del bosque. Unasylva, 238(62), 39-46.

Mora, R., Vahrson, W.-G. and Mora, S. (1992) 'Mapa de amenaza de deslizamientos, Valle Central, Costa Rica', CEPREDENAC. Ciudad de Guatemala, Guatemala.

Mora, S., \& Vahrson, W. (1994). Macrozonation Methodology for Landslide Hazard Determination. Bulletin of the Association of Engineering Geologists, 31(1), 48-58.

SGN. (2002). Carta Geológica-Minera 1:250,000.

Sieron, K. (2018). Caracterización del área de estudio. Informe interno de Proyecto "ECOPICS". SMN. (2018). Información climatológica por estado. Base de datos díarios dEl Estado de Puebla y Veracruz.

Quesada, A. and Feoli, S. (2018) 'Comparación de la Metodología Mora-Vahrson y el Método Morfométrico para Determinar Áreas Susceptibles a Deslizamientos en la Microcuenca del Río Macho, Costa Rica', Revista Geográfica de América Central, 60, pp. 17-45. Available

at:

http://revistas.una.ac.cr/index.php/geografica/article/download/10677/13252?inline=1.

Van Zuidam, R. (1985). Terrain analysis and classification using aerial photographs. International Institute for aereal survey and earth sciences, 2.

Zimbelman, D. R. et al. (2004) 'Stratovolcano stability assessment methods and results from Citlaltépetl , Mexico', pp. 66-79. doi: 10.1007/s00445-003-0296-8. 